\title{
Biological Drivers of Wilms Tumor Prognosis and Treatment
}

\author{
Hannah M. Phelps ${ }^{1, *(D)}$, Saara Kaviany ${ }^{2}$, Scott C. Borinstein ${ }^{2}\left(\mathbb{D}\right.$ and Harold N. Lovvorn, III ${ }^{3}$ (D) \\ 1 Vanderbilt University School of Medicine, Vanderbilt University, Nashville, TN 37232, USA \\ 2 Department of Pediatrics, Division of Pediatric Hematology-Oncology, Vanderbilt University Medical \\ Center Nashville, TN 37232, USA; saara.kaviany@vumc.org (S.K.); scott.c.borinstein@vumc.org (S.C.B.) \\ 3 Department of Pediatric Surgery, Vanderbilt University Medical Center Nashville, TN 37232, USA; \\ harold.lovvorn@vumc.org \\ * Correspondence: hannah.m.phelps@vanderbilt.edu; Tel.: +1-314-307-3825
}

Received: 29 September 2018; Accepted: 18 October 2018; Published: 26 October 2018

\begin{abstract}
Prior to the 1950s, survival from Wilms tumor (WT) was less than $10 \%$. Today, a child diagnosed with WT has a greater than $90 \%$ chance of survival. These gains in survival rates from WT are attributed largely to improvements in multimodal therapy: Enhanced surgical techniques leading to decreased operative mortality, optimization of more effective chemotherapy regimens (specifically, dactinomycin and vincristine), and inclusion of radiation therapy in treatment protocols. More recent improvements in survival, however, can be attributed to a growing understanding of the molecular landscape of Wilms tumor. Particularly, identification of biologic markers portending poor prognosis has facilitated risk stratification to tailor therapy that achieves the best possible outcome with the least possible toxicity. The aim of this review is to (1) outline the specific biologic markers that have been associated with prognosis in WT and (2) provide an overview of the current use of biologic and other factors to stratify risk and assign treatment accordingly.
\end{abstract}

Keywords: Wilms tumor; nephroblastoma; tumor biology; biomarkers; therapy

\section{Introduction}

Wilms tumor (WT), also called Wilms' tumor or nephroblastoma, represents approximately $95 \%$ of childhood kidney tumors, with an incidence of approximately 7 cases per million children aged 15 years or younger [1]. WTs are classified among the embryonal tumors of childhood given the histologic and molecular resemblance to the embryonic kidney. Survival from WT now exceeds $90 \%$ at 5 years, in large part due to an increasing understanding of WT biology and the identification of molecular markers that associate with treatment resistance and poor prognosis. Decades of cooperative, multi-disciplinary trials have achieved these high overall survival rates, and research efforts are now aimed at minimizing toxic therapies while maintaining excellent survival. Additional research efforts have focused on the more lethal subsets of WT, including relapsed disease, anaplastic histology, and bilateral disease that collectively account for most treatment morbidity and cancer-related deaths. Wilms tumors historically were risk stratified by histology, defined as favorable (FHWT) or unfavorable histology (UHWT or diffusely anaplastic WT; 8\%) [2].

Centralized research consortia have enabled large-scale analysis of this relatively rare disease. The two primary organizations from which treatment plans emerge are the Children's Oncology Group (COG; formerly the NWTS) in North America and the International Society of Pediatric Oncology (SIOP) in Europe. Protocols established through both cooperative groups incorporate surgical resection of the tumor (either total or partial nephrectomy), chemotherapy, and radiotherapy when indicated. However, the timing and coordination of therapy differs between groups. COG protocols dictate 
upfront nephrectomy when possible, advocating earlier histologic diagnosis and more accurate staging information through lymph node assessment that together guide adjuvant therapy [1]. Upfront resection also provides an opportunity to minimize or avoid exposure to chemotherapy in certain subgroups. SIOP protocols, in contrast, recommend neoadjuvant chemotherapy without biopsy, followed by delayed resection. Adjuvant therapy is then tailored to histologic tumor features. This approach is associated with fewer cases of intra-operative tumor spill and lower post-operative tumor stage [3]. This review will focus on efforts by the COG, acknowledging that the work of each group is invaluable to the other.

Under COG protocols, WT stage is based on completeness of resection, perioperative rupture of tumor capsule, lymph node spread, and distant metastases (Table 1). Further risk stratification tailors therapy based on clinical (i.e., stage, patient age, tumor weight, and completeness of lung nodule response after six weeks of chemotherapy) and biologic (i.e., histology and loss of heterozygosity at $1 \mathrm{p}$ and $16 q$ ) features. Risk stratification according to histology and biology therefore helps to decrease exposure to toxic therapies for more favorable tumor features while maintaining excellent survival rates. The goal of this review is to summarize the key biological factors that impact current or potential future treatment regimens for WT.

Table 1. Wilms tumor stages according to the Children's Oncology Group.

\begin{tabular}{|c|c|}
\hline Stage & Criteria \\
\hline Stage I & $\begin{array}{l}\text { Confined to kidney } \\
\text { Complete excision with renal capsule intact and negative resection margins } \\
\text { Lymph nodes negative for Wilms tumor spread }\end{array}$ \\
\hline Stage II & $\begin{array}{l}\text { Regional extension beyond kidney capsule, but confined to flank } \\
\text { May include: } \\
\text { Tumor penetration through capsule but confined to Gerota's fascia } \\
\text { Infiltration into renal vein } \\
\text { Complete excision with negative resection margins } \\
\text { Lymph nodes negative for Wilms tumor spread }\end{array}$ \\
\hline Stage III & $\begin{array}{l}\text { Residual tumor, but confined to abdomen } \\
\text { May include: } \\
\text { Regional lymph node involvement } \\
\text { Peritoneal contamination: } \\
\text { Biopsy } \\
\text { Pre- or intraoperative tumor rupture } \\
\text { Tumor growth through peritoneal surface } \\
\text { Positive resection margins }\end{array}$ \\
\hline Stage IV & Distant metastases: Lung, liver, bone, brain \\
\hline Stage V & Involvement of bilateral kidneys at diagnosis \\
\hline
\end{tabular}

\section{Genomic and Molecular Alterations with Prognostic Significance in WT}

\subsection{Chromosome 11}

Chromosome 11p harbors two distinct WT suppressor genes, WT1 and WT2. Early steps to understand WT biology were driven by the observation that children with WAGR syndrome (Wilms tumor, Aniridia, Genitourinary Malformations, and Retardation) had a constitutional deletion of 11p13. The specific gene, WT1, was isolated by identifying the minimum deletion region of that locus [5]. WT1 is a transcriptional regulator likely involved in coordinating differentiation of genitourinary tissues, though its exact function in kidney development remains incompletely characterized [6]. WT1 mutations occur in only about $15 \%$ of sporadic WT, suggesting the involvement of other key genes in tumorigenesis. Loss of both copies of WT1 has been described as a hallmark of WT development 
in WAGR and other constitutional genetic conditions, such as Denys-Drash and Frasier Syndromes, suggesting loss of tumor suppressor function.

A second gene, WT2, located at 11p15, is altered at a much higher frequency in WT ( 70\%) [3]. Linkage analysis has mapped the gene for Beckwith-Wiedemann Syndrome (BWS, another WT predisposition syndrome) to the $11 \mathrm{p} 15$ locus, and $11 \mathrm{p} 15$ is now recognized as a region of genomic imprinting. In normal development, the maternal allele for IGF2 and the paternal allele for $H 19$ are silenced at this locus. Loss of imprinting (LOI) and subsequent activation of the normally silenced maternal IGF2 (insulin-like growth factor 2) allele result in the most common genetic alteration in WT, occurring in $30-50 \%$ of sporadic WT $[7,8]$. Paternal uniparental disomy, whereby one chromosome is lost and the remaining chromosome is duplicated, may also lead to copy neutral loss of heterozygosity (LOH) at 11p15 and subsequent overexpression of IGF2. It is thought in the embryonic kidney that if biallelic methylation of $11 \mathrm{p} 15$ occurs before induction, high levels of IGF2 cause preferential proliferation of the metanephric mesenchyme, impair development of the nephron, and yield persistence of these rogue progenitors in the form of nephrogenic rests (NR), the putative precursor lesion of WT. This event hypothetically results in intralobar NR. If the biallelic methylation of $11 \mathrm{p} 15$ occurs after induction, high levels of IGF2 prevent terminal epithelial differentiation, resulting in perilobar NR [9]. It is thought that intralobar NR occur at an earlier stage in renal development than perilobar NR. Notably, WT1 alterations are also associated with development of intralobar NR [10]. As noted, NR represent the precursor lesion of WT, but a second genetic event within this population of stem cells likely initiates development of WT.

Importantly, the WT1 mutation and 11p15 LOH or LOI are thought to drive distinct pathogenetic mechanisms for the development and/or progression of WT; however, these events are not necessarily independent given the close proximity of the 11p13 and 11p15 loci. In one study, all patients with WT1 mutations also had 11p15 LOH, yet 11p15 LOH was identified without WT1 mutations in a proportion of cases. Therefore, $11 \mathrm{p} 15$ is likely the more sensitive prognostic indicator [11].

\subsection{Loss of Heterozygosity at $1 p$ and $16 q$}

Early studies utilizing LOH mapping identified chromosomes $1 \mathrm{p}$ and $16 \mathrm{q}$ as regions of interest in WT genetics, with $17 \%$ of tumors harboring $\mathrm{LOH}$ at $16 \mathrm{q}$ and $12 \%$ at $1 \mathrm{p}[12,13]$. Concurrent $\mathrm{LOH}$ at both loci was associated with adverse prognosis among favorable histology WT (FHWT) [12]. Since then, multiple studies in North America and Europe have suggested that LOH at $1 \mathrm{p}$ and/or $16 \mathrm{q}$ associates with relapse and overall poor prognosis [14-17]. Most recently, the NWTS-5 clinical trial demonstrated that combined tumor-specific LOH of chromosomes 1p and 16q in patients with Stage III and IV disease was associated with inferior 4-year event-free survival (EFS) and overall survival (OS) in a cohort of greater than 1700 patients [18]. Based on these findings, the COG clinical trials AREN0532 and AREN0533 intensified chemotherapy for patients with FHWT that demonstrated LOH at $1 \mathrm{p}$ and 16q. To date, combined $\mathrm{LOH}$ at $1 \mathrm{p} / 16 \mathrm{q}$ is the only molecular marker employed in risk stratification. Though it appears to be a sensitive marker for predicting relapse, $\mathrm{LOH}$ at $1 \mathrm{p}$ and $16 \mathrm{q}$ is not broadly specific, as this combination was present in only $9.4 \%$ of relapsed tumors. Overall, combined LOH at $1 \mathrm{p}$ and $16 \mathrm{q}$ is present in only $5 \%$ of FHWT [18].

Efforts to identify a mechanistic explanation for these observations demonstrated that $16 \mathrm{q}$ loss associated interestingly with LOI at 11p15. The authors speculated that haploinsufficiency of the CCCTC-Binding Factor (CTCF) gene, which maps to 16q22 and codes for an insulator protein that regulates IGF2 imprinting, is the mechanism behind this association. Indeed expression of CTCF in tumors with $\mathrm{LOH}$ at 16q was half that of tumors with normal chromosome 16 [19].

\subsection{Copy Number Gain at 1q}

Copy number gain of chromosome $1 \mathrm{q}$ is a commonly observed genetic abnormality in WT and is present in approximately $30 \%$ of tumors [20]. After several smaller, retrospective studies suggested a correlation between 1q gain and tumor recurrence, the Children's Cancer and Leukaemia Group, 
NWTS, and SIOP independently confirmed poorer EFS and OS in both pre-treated and untreated patients with 1q gain in larger cohorts [21-26]. Data collected through the NWTS-5 clinical trial was used to assess the prognostic significance of 1q gain in FHWT with sufficient power to detect survival differences within stage groups. Among all stages, 8-year EFS was 77\% (95\% CI, 72-81\%) for patients with 1q gain, and OS was $88 \%$ (95\% CI, 83-91\%). For patients without 1q gain, 8-year EFS was $90 \%$ (95\% CI, 88-92\%), and OS was 96\% (95\% CI, 94-97\%) [20]. No significant difference in histologic predominance based on presence or absence of 1q gain was observed. Stage IV disease was more common among patients with 1q gain compared to those without $(18.3 \%$ vs. $9.4 \%, p<0.001)$, suggesting that the abnormality is associated with a more aggressive and malignant phenotype. In a stage-by-stage comparison, EFS was significantly poorer for patients with 1q gain and Stage I, III, and IV disease [20]. 1q gain was not independent of $1 p$ and 16q loss, which is not surprising given that isochromosome $1 \mathrm{q}$ and translocations between chromosomes 1 and 16 are common genetic abnormalities in WT. Both alterations can cause loss of $1 p$ or 16q and gain of 1q [20]. It remains unclear which abnormality and which relevant gene(s) accounts for the poorer outcomes in patients with these genetic abnormalities. Of note, 1q gain has been associated with poor outcomes in other embryonal tumors including neuroblastoma, pediatric ependymoma, medulloblastoma, Ewing sarcoma, as well as other adult tumors [27-30].

\subsection{Alterations at $17 p$}

TP53 mutations have been identified in 50-86\% of anaplastic WT [31,32]. Presence of p53 in anaplastic regions but not in non-anaplastic regions of the same tumor suggests that anaplasia stems from a clonal event that takes place in a FHWT. A recent COG study using patient samples collected in NWTS along with the TARGET Data Matrix demonstrated TP53 mutations in $48 \%$ of patients with diffuse anaplastic WT (DAWT) $(n=118)$ [31,33]. An additional $11 \%$ without mutations had loss of 17p13 (TP53 locus). TP53 abnormalities do not appear to associate with stage of DAWT but are associated with significantly worse disease-free and overall survival (OS) for patients with Stage III or IV DAWT [31]. The association between DAWT and TP53 mutation has been substantiated in multiple other studies $[32,34,35]$. The authors speculated that the low degree of anaplasia in most tumors lacking TP53 mutation and/or copy loss indicate that detection of a TP53 abnormality reflects the "burden of anaplasia. The "burden of anaplasia" may not have a survival impact on Stage I and II anaplastic tumors if completely resected as compared to Stage III and IV anaplastic disease, which is at higher risk of residual or systemic tumor after surgery [31]. Another recent study sought to characterize TP53 status in a cohort of fatal WT cases. TP53 alterations (detected by sequencing, copy number, and/or protein expression) were detected in 52\% (45/86) of fatal WT [35]. TP53 alterations were detected in $97 \%$ (29/30) of tumors with DAWT but were also present in $26 \%$ of non-anaplastic tumors. Of note, many of the non-anaplastic tumors partially fulfilled anaplastic criteria (e.g., nuclear unrest). These results, coupled with the observation that p53 positivity on IHC was not limited to anaplastic cells, but expanded areas surrounding anaplasia, suggest that TP53 status could also be a screening tool for tumors with partial phenotypes $[35,36]$.

\subsection{Loss of Heterozygosity at $4 q$ and $14 q$}

In addition to alterations at the TP53 locus, molecular profiling has demonstrated significant associations between anaplastic histology and loss of $4 q$ and 14q [34]. Specific candidate genes involved in WT pathogenesis at these latter loci have not been identified yet, and the significance of these genomic alterations remains unknown.

\subsection{MYCN Amplification}

Genomic amplification of the $M Y C N$ gene has repeatedly been described in WT as well as other embryonal tumors, most commonly in neuroblastoma [37-40]. Overexpression of MYCN in WT has been identified as a potentially prognostic feature [34,41,42]. Interestingly, MYCN gain was 
present in higher proportion $(>30 \%$ ) among a cohort of pre-treated anaplastic tumors compared with a parallel study analyzing a mixed cohort of anaplastic tumors (which included tumors that were not pre-treated). This suggests that $M Y C N$ gain could confer treatment resistance [34,43]. Notably, MYCN gain is not limited to anaplastic WT, and its association with poorer relapse-free and overall survival is independent of histology [42,44]. The P44L mutation has been identified as a potentially activating mutation leading to MYCN gain in WT [44].

\section{7. $\mathrm{LOH}$ at $11 q$}

Among a cohort of 225 tumors treated on the SIOP protocol, 19.6\% (44) demonstrated allele loss on chromosome arm 11q. Frequency of $\mathrm{LOH}$ at 11q was 3-4 times higher among mixed type and diffuse anaplastic tumors compared to favorable histology tumors. Loss of the entire long arm of chromosome 11 was associated with higher rates of relapse and death [16]. Other studies have also demonstrated a correlation between $\mathrm{LOH}$ at 11q and anaplasia, tumor recurrence, and death, indicating that this region is likely prognostically relevent [15].

\section{Current Risk Assignment and Treatment Strategies for Wilms Tumor}

This next section will focus on how these biologic data have stratified the treatment approach to WT patients having specific clinical, histologic and molecular alterations. The overarching objective of these risk strata is to tailor therapy that is less toxic for more favorable WT cases and intensified for those patients having a higher risk of treatment failure.

\subsection{Very Low Risk}

Very low risk Wilms Tumor (VLRWT) is defined as age less than 2 years, favorable histology, small tumor $(<550 \mathrm{~g})$ confined to the renal capsule and completely resected. This definition first emerged out of NWTS-5 study after analyses showed that adjuvant chemotherapy did not improve survival in certain subsets of low risk patients [45]. The subsequent attempt to eliminate adjuvant chemotherapy among patients meeting very low risk criteria resulted in early termination due to EFS $(86.5 \%)$ at the 3-year interim-analysis, which was inferior to the set stopping point (90\%) [46]. However, later analysis revealed high salvage rates with three-drug chemotherapy (vincristine, dactinomycin, and doxorubicin) and site-specific radiotherapy, resulting in an excellent overall survival [47].

Using this definition for VLRWT, the subsequent COG Trial AREN0532 enrolled 116 patients for nephrectomy-only therapy. At four years, 12 patients had relapsed (4-year EFS 89.7\%), and OS was $100 \%$. Biologic features were evaluated as potential indicators of children at risk for relapse. 1q gain, 1p loss, and/or 16q loss and mutation of WT1 were not associated with relapse; however, 11 p15 methylation status, particularly LOH and LOI, was associated with relapse in this otherwise favorable group [48]. The authors concluded that the observation-only approach to VLRWT potentially evades chemotherapeutic drug toxicity and complications from central lines, yet exposes relapsed patients to anthracyclines and radiation, which may have been avoided if the standard adjuvant vincristine/dactinomycin had been delivered. While $11 \mathrm{p} 15$ status is a potential predictor of relapse, with a $20-25 \%$ risk of recurrence in patients with $\mathrm{LOI} / \mathrm{LOH}$ compared to a $3 \%$ risk in those with no $\mathrm{LOI} / \mathrm{LOH}$, most patients with $11 \mathrm{p} 15$ methylation abnormalities are predicted not to relapse [48]. The WT1 mutation has also been associated with relapse among VLRWT treated with nephrectomy-only and could be applied instead of or in addition to 11p15 methylation status to predict risk of relapse in these patients [11]. Based on the results of AREN0532, the current treatment strategy for VLRWT is nephrectomy followed by observation (Table 2) [48]. Given these encouraging findings, future studies are under consideration to increase the tumor weight limit and expand the age range included in the definition of VLRWT [48]. 
Table 2. Current risk stratification, treatment regimens, and outcomes.

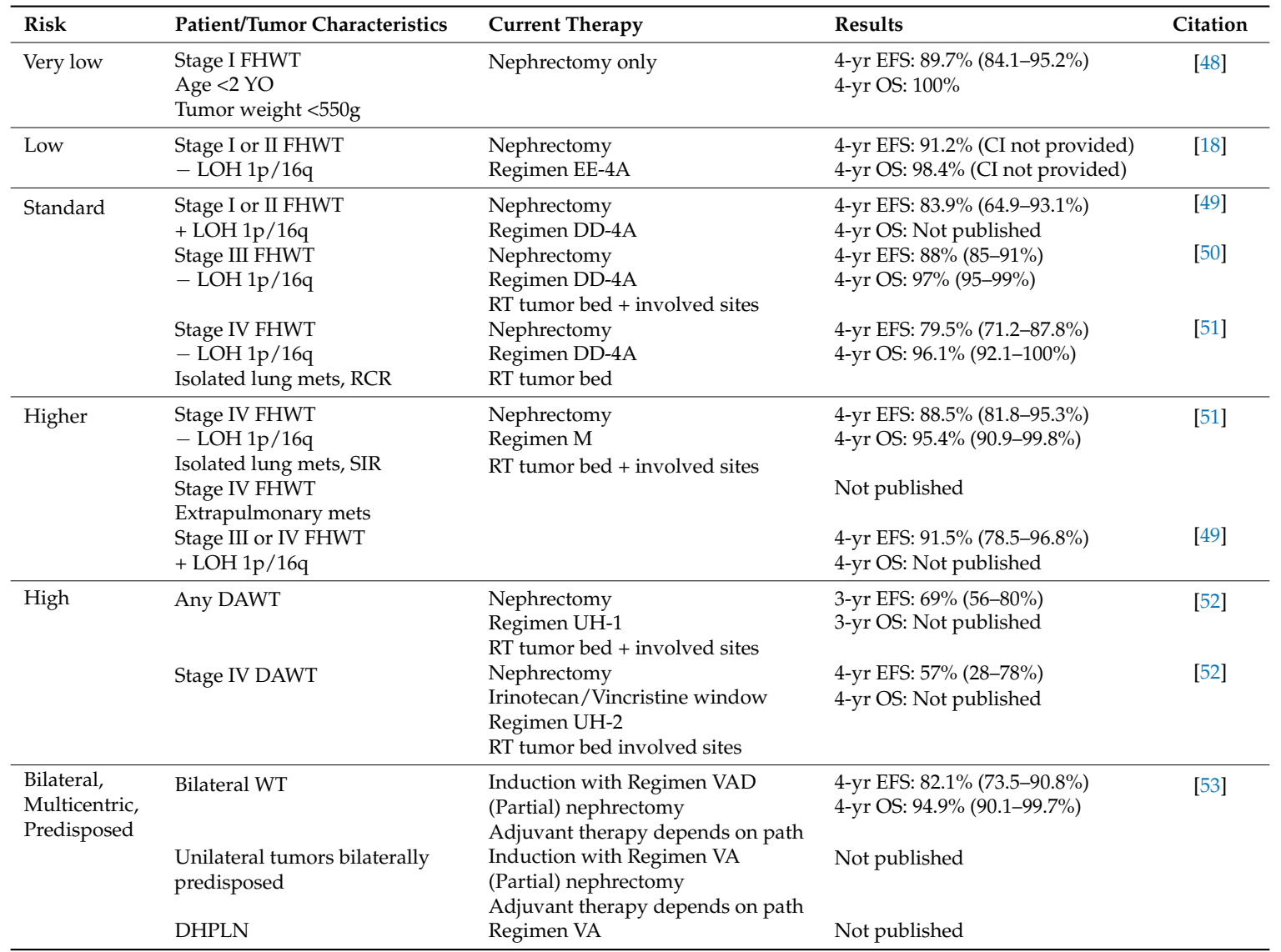

WT, Wilms tumor; FHWT, favorable histology Wilms tumor; DAWT, diffuse anaplastic Wilms tumor; LOH, loss of heterozygosity; EFS, event-free survival; OS, overall survival; RT, radiation therapy; RCR, rapid complete response; SIR, slow incomplete response; DHPLN, diffuse hyperplastic perilobar nephroblastomatosis; REGIMEN EE-4A = vincristine and dactinomycin $\times 16$ weeks; REGIMEN DD-4A = dactinomycin, vincristine, and doxorubicin $\times 24$ weeks; REGIMEN M = vincristine, dactinomycin, doxorubicin, cylophosphamide, etoposide $\times 24$ weeks; REGIMEN UH- $1=$ alternating cyclophosphamide/carboplatin/etoposide and vincristine/doxorubicin/cyclophosphamide $\times 30$ weeks; WINDOW THERAPY = vincristine and irinotecan $\times 6$ weeks (assessment of response at 3 weeks); REGIMEN UH-2= cyclophosphamide/carboplatin/etoposide and vincristine/doxorubicin/cyclophosphamide + irinotecan; REGIMEN $\mathrm{VAD}=$ vincristine, dactinomycin, doxorubicin; REGIMEN VA = vincristine, dactinomycin .

\subsection{Low Risk}

\section{Stage I and II FHWT without $\mathrm{LOH}$ at $1 \mathrm{p} / 16 \mathrm{q}$}

WT patients having Stage I or II disease and not meeting very low risk criteria are treated with upfront nephrectomy, when feasible, followed by adjuvant chemotherapy with vincristine and dactinomycin (Regimen EE-4A, Table 2). This low risk category has not been evaluated on a separate AREN trial to date, but NWTS-5 data showed that this group attained 4-year EFS and OS of $91.2 \%$ and $98.4 \%$, respectively [18].

\subsection{Standard Risk}

\subsubsection{Stage I and II FHWT with LOH at $1 p / 16 q$}

Prospective, large-scale analysis through NWTS-5 identified adverse prognosis among FHWT patients having $\mathrm{LOH}$ at $1 \mathrm{p} / 16 \mathrm{q}$. These data prompted intensification of therapy for patients with this combined genetic marker. Under AREN0532, patients with Stage I or II FHWT demonstrating concurrent $\mathrm{LOH}$ at $1 \mathrm{p}$ and $16 \mathrm{q}$ were provided with a standard risk treatment regimen in place of the low risk regimen used for Stage I and II FHWT without LOH 1p/16q. The standard risk regimen 
consists of upfront nephrectomy when possible followed by Regimen DD-4A (doxorubicin, vincristine, and dactinomycin) without radiation therapy (Table 2). EFS for this subset of patients was $83.9 \%$ (64.9-93.1\%), and OS has not been published at this time [49].

\subsubsection{Stage III FHWT without $\mathrm{LOH}$ at $1 \mathrm{p} / 16 \mathrm{q}$}

The current approach to treating Stage III FHWT, carried over from NTWS-5, includes upfront nephrectomy, when feasible, followed by Regimen DD-4A and abdominal radiation therapy (Table 2). The AREN0532 study demonstrated continued excellent EFS (88\% [95\% CI, 85-91\%]) and OS (97\% [95\% CI, 95-99\%]) for Stage III FHWT without combined LOH 1p/16 treated with this protocol [50]. The study additionally sought to identify clinical, pathologic, and molecular features associated with worse prognosis that might potentially be used for future risk stratification. Poorer EFS was associated with positive lymph node status and $\mathrm{LOH}$ at $1 \mathrm{p}$ or 16q. Patients with positive lymph nodes and $\mathrm{LOH}$ at $1 p$ or $16 q$ had EFS of only $74 \%$. Gross residual disease and peritoneal implants were not associated with poorer EFS [50]. 1q gain status was not available for the AREN0532 cohort, but future therapeutic protocols under consideration include assessment of $1 \mathrm{q}$ gain in addition to $\mathrm{LOH}$ at $1 \mathrm{p} / 16 \mathrm{q}$ so that doxorubicin might be omitted in patients that lack these risk factors [50].

3.3.3. Stage IV FHWT with Isolated Lung Metastases Responding Completely to Chemotherapy but without $\mathrm{LOH}$ at $1 \mathrm{p} / 16 \mathrm{q}$

Under former NWTS protocols, all patients with metastatic lung disease received lung radiation. Recent changes in treatment protocols have focused on reducing the number of patients exposed to lung radiation without compromising EFS and OS. On AREN0533, the need for lung radiation in patients with Stage IV FHWT having isolated lung metastases was determined by $\mathrm{LOH}$ at $1 \mathrm{p} / 16 \mathrm{q}$ as well as lung nodule response to chemotherapy (Table 2). Data from NWTS-5 demonstrated the association between combined $\mathrm{LOH}$ at $1 \mathrm{p} / 16 \mathrm{q}$ and adverse prognosis, serving as the rationale to incorporate $\mathrm{LOH}$ at $1 \mathrm{p} / 16 \mathrm{q}$ as a factor to stratify further risk among Stage IV FHWT [18]. The addition of lung nodule response to the risk stratification scheme for AREN0533 was derived from SIOP protocols, which dictate 6 weeks of pre-nephrectomy vincristine/dactinomycin/doxorubicin to determine next steps in therapy. After 6 weeks of treatment, if a complete response (as defined by chest computerized tomography) is achieved without evidence for remaining lung nodules, either from chemotherapy or surgical resection, lung radiation is excluded from the subsequent treatment regimen. With this strategy, the SIOP has reported 5-year EFS of $77 \%$ and OS of $87 \%$, comparable to the $76 \%$ EFS reported by NWTS-5 [54,55].

Based on the protocol outlines in AREN0533 and the encouraging SIOP findings, currently, patients with Stage IV FHWT but without $\mathrm{LOH}$ at $1 \mathrm{p} / 16 \mathrm{q}$ who demonstrate a complete response after 6 weeks of DD-4A therapy continue with Regimen DD-4A regimen and do not receive lung radiation. However, patients without a complete lung nodule response or with $\mathrm{LOH}$ at $1 \mathrm{p} / 16 \mathrm{q}$ are transitioned to the higher risk stratum (see 3.3.1 below). Among 292 patients presenting with isolated lung metastases enrolled on AREN0533, 133 (46\%) demonstrated complete response after 6 weeks of DD-4A. Four-year EFS was $79.5 \%$ (95\% CI, 71.2-87.8\%) and OS was $96.1 \%$ (95\% CI, 92.1-100\%) for patients without LOH $1 \mathrm{p} / 16 \mathrm{q}$ and showing complete response. Initial lung radiation was avoided in approximately $40 \%$ of patients. A post hoc analysis of prognostic significance of 1q gain was performed, which associated significantly with poorer 4-year EFS and a trend toward poorer OS among patients demonstrating otherwise a complete pulmonary response to DD-4A [51]. This result suggests a role for assessing 1q gain in this stratum to identify patients who may not be good candidates for omission of lung radiation despite the resolution of lung nodules with chemotherapy. 


\subsection{Higher Risk}

\subsubsection{Stage III or IV FHWT with LOH 1p/16q}

Again, in response to poorer prognosis among patients with LOH 1p/16q established in NWTS-5, AREN0533 augmented therapy for patients with Stage III or IV FHWT and LOH 1p/16, assigning these cases to the intensified protocol defined as Regimen M (incorporating cyclophosphamide and etoposide to vincristine, dactinomycin, and doxorubicin) in addition to radiation therapy (Table 2). Preliminary results published in abstract form indicate that 4-year EFS for patients treated under this protocol was $91.5 \%$ (95\% CI, 78.5-96.8\%), which is significantly better than those historically treated with Regimen DD-4A [49].

3.4.2. Stage IV FHWT without $\mathrm{LOH}$ at $1 \mathrm{p} / 16 \mathrm{q}$ but with Isolated Lung Metastases Responding Incompletely to Chemotherapy

Patients without LOH 1p/16q and with isolated lung metastases initiated on DD-4A who do not exhibit complete pulmonary response are converted to the higher risk stratum to receive Regimen M. Four-year EFS and OS for patients with incomplete response was 88.5\% (95\% CI, 81.8-95.3\%) and 95.4\% (95\% CI, 90.9-99.8\%), respectively. No significant association was detected between 1q gain and EFS or OS in patients with incomplete response [51].

\subsubsection{Stage IV FHWT with Extrapulmonary Metastases}

Stage IV FHWT with extrapulmonary metastases is currently treated with upfront nephrectomy, if feasible, followed by Regimen $\mathrm{M}$ and radiation therapy to the tumor bed and involved sites. Of 349 patients with Stage IV FHWT assessed for inclusion in AREN0533, 52 patients had extrapulmonary metastases. Outcomes for this group are yet to be described in detail.

\subsection{High Risk}

Tumors demonstrating diffuse anaplasia (DAWT) are defined as high risk. As previously described, several biologic markers, including TP53 mutation, $\mathrm{LOH}$ at $4 \mathrm{q}$ and $14 \mathrm{q}$, and $\mathrm{LOH}$ at 11q, have been shown to associate with DAWT. High risk, DAWT is currently treated with Regimen UH-1, which incorporates cyclophosphamide, carboplatin, etoposide, vincristine, and doxorubicin. Under AREN0321, patients with metastatic (Stage IV) DAWT also had the option to participate in a window study to assess the antitumor activity of vincristine and protracted-schedule irinotecan against metastatic (Stage IV) DAWT. The results of the phase II window study indicate preliminarily that among 14 participants with Stage IV DAWT, 11 had partial response and 3 had progressive disease with treatment. Patients with a partial response had vincristine and irinotecan incorporated into Regimen UH-1 (called Regimen UH-2). Notably, two of the three non-responders also did not respond to additional treatment [56]. Based on these results, continued monitoring of the efficacy of neoadjuvant vincristine and irinotecan for metastatic DAWT is underway. Among Stage II-IV DAWT treated with Regimen UH-1/UH-2, three-year EFS was 69\% (95\% CI, 56-80\%), representing improved EFS from patients treated on Regimen I (lacking carboplatin) under NWTS. However, toxicity was also increased with Regimen UH-1/UH-2 as three patients (4.5\%) died from treatment toxicity [52].

\subsection{Bilateral, Multicentric, or Bilaterally-Predisposed Unilateral Wilms Tumor}

\subsubsection{Bilateral WT}

Bilateral Wilms Tumor has historically been associated with poor outcomes (4-year EFS $56 \%$ according to NWTS-5) [53]. The current regimen for treating bilateral WT is derived from AREN0534 with the primary goal of improving EFS and OS while preserving renal parenchyma. The protocol starts with intensive neoadjuvant chemotherapy (VAD Regimen; vincristine, dactinomycin, and doxorubicin) and mandates definitive surgery by 12 weeks. Adjuvant therapy is modified based on the histologic 
response. Among 242 patients enrolled on AREN0533 for bilateral WT, 21.1\% had a genetic predisposition syndrome. Among 189 patients treated and evaluated, four-year EFS and OS were 82.1\% (95\% CI,73.5-90.8\%) and 94.9\% (95\% CI, 90.1-99.7\%), respectively. Definitive surgery was performed by 12 weeks in $84 \%$ of patients, of which $39 \%$ were successfully treated with bilateral nephron-sparing surgery [53]. Further studies are needed to assess the role of genetic markers in predicting risk of relapse.

\subsubsection{Genetically Predisposed WT and Diffuse Hyperplastic Perilobar Nephroblastomatosis (DHPLN)}

Patients who develop WT in the context of a genetic predisposition syndrome (e.g., WAGR, Denys-Drash, BWS) are at high risk for metachronous or multicentric tumors through 8 years of age. Preservation of renal parenchyma is of utmost importance in this patient population. To this end, current treatment protocols involve 2-drug induction with vincristine/dactinomycin with the goal of facilitating partial, rather than complete, nephrectomy. Data collected under AREN0533 for this specific treatment strategy has not yet been published.

A similar chemotherapy regimen with vincristine/dactinomycin has been utilized for the goal of preventing development of WT from DHPLN. Results of this arm of AREN0533 have also not yet been published.

\section{Conclusions}

Significant progress has been made to identify indicators of poor prognosis with WT and to adjust treatment algorithms accordingly. So far, $\mathrm{LOH}$ at $1 \mathrm{p} / 16 \mathrm{q}$ is the only biologic marker included in WT risk stratification, but emerging evidence has identified several additional markers of poor prognosis, including mutations of WT1, 1q gain, alterations at 17p, LOH at $4 \mathrm{p}$ and $14 \mathrm{q}, M Y C N$ amplification, and $\mathrm{LOH}$ at 11q. Incorporation of additional biologic markers, particularly 1q gain, into treatment protocols can be expected in the coming years. As one example to reduce toxic exposures using tumor-specific biology moving forward, doxorubicin might be omitted in Stage III FHWT without LOH $1 \mathrm{p} / 16 \mathrm{q}$ and 1q gain (see Section 3.3.2), although such a proposal would require evaluation through a rigorous cooperative trial to determine no change in adverse event frequency while mitigating early and late treatment side effects. Additional efforts are underway to identify differences in tumor genetics based on race and ethnicity, which could provide further insight into risk stratification and allow for even more personalized treatment strategies.

As risk stratification becomes more dependent on biological markers, it is important to consider the reliability of analytical tests and methods employed to identify these factors. WT are often huge at resection and show regions of histologic variability regarding cellular predominance (i.e., content of blastema, epithelia, and stroma vary) and necrosis. The degree to which a single, small sample (usually $\sim 250 \mathrm{mg}$ in size) can reliably represent an entire tumor has not been adequately explored in WT or other large, embryonal tumors. Recently, one study demonstrated that certain genetic markers thought to represent earlier events in tumor development, such as LOH at 11p15, are uniformly detected in WT specimens. However, later genetic events, such as 1q gain are variably detected in different samples derived from the same tumor. The authors estimated that at least 3 samples per tumor should be analyzed to detect $>95 \%$ of cases of 1q gain [57]. Studies demonstrating the prognostic significance of genetic markers in WT largely relied on the assumption that one sample is sufficient for detecting the marker of interest. In light of these new data, the currently accepted markers of poor prognosis must be verified in studies applying more rigorous tumor sampling. Further, multiple samples of a tumor specimen should be assessed clinically to determine risk stratification.

The ability to risk stratify WT based on patient and tumor characteristics has resulted in significant progress in improving survival while reducing exposure to toxic therapies. At the same time, these stratifications have created multiple subgroups of patients with numbers too small to power clinical trials. Thus, there is growing need for collaboration among institutions, nationally and internationally [58]. 
Funding: This project received no external funding.

Conflicts of Interest: The authors declare no conflict of interest.

\section{References}

1. Pizzo, P.A.; Poplack, D.G.; Adamson, P.C.; Blaney, S.M.; Helman, L. Principles and Practice of Pediatric Oncology, 7th ed.; Wolters Kluwer Health: Philadelphia, PA, USA, 2016.

2. Faria, P.; Beckwith, J.B.; Mishra, K.; Zuppan, C.; Weeks, D.A.; Breslow, N.; Green, D.M. Focal versus diffuse anaplasia in Wilms tumor-New definitions with prognostic significance: A report from the national Wilms tumor study group. Am. J. Surg. Pathol. 1996, 20, 909-920. [CrossRef] [PubMed]

3. Brok, J.; Treger, T.D.; Gooskens, S.L.; van den Heuvel-Eibrink, M.M.; Pritchard-Jones, K. Biology and treatment of renal tumours in childhood. Eur. J. Cancer 2016, 68, 179-195. [CrossRef] [PubMed]

4. Davidoff, A.M. Wilms tumor. Adv. Pediatr. 2012, 59, 247-267. [CrossRef] [PubMed]

5. Call, K.M.; Glaser, T.; Ito, C.Y.; Buckler, A.J.; Pelletier, J.; Haber, D.A.; Rose, E.A.; Kral, A.; Yeger, H.; Lewis, W.H.; et al. Isolation and characterization of a zinc finger polypeptide gene at the human chromosome 11 Wilms' tumor locus. Cell 1990, 60, 509-520. [CrossRef]

6. Grubb, G.R.; Yun, K.; Williams, B.R.; Eccles, M.R.; Reeve, A.E. Expression of WT1 protein in fetal kidneys and Wilms tumors. Lab. Invest. 1994, 71, 472-479. [PubMed]

7. Bjornsson, H.T.; Brown, L.J.; Fallin, M.D.; Rongione, M.A.; Bibikova, M.; Wickham, E.; Fan, J.B.; Feinberg, A.P. Epigenetic specificity of loss of imprinting of the IGF2 gene in Wilms tumors. J. Natl. Cancer Inst. 2007, 99, 1270-1273. [CrossRef] [PubMed]

8. Ogawa, O.; Eccles, M.R.; Szeto, J.; McNoe, L.A.; Yun, K.; Maw, M.A.; Smith, P.J.; Reeve, A.E. Relaxation of insulin-like growth factor II gene imprinting implicated in Wilms' tumour. Nature 1993, 362, 749-751. [CrossRef] [PubMed]

9. Gadd, S.; Huff, V.; Huang, C.C.; Ruteshouser, E.C.; Dome, J.S.; Grundy, P.E.; Breslow, N.; Jennings, L.; Green, D.M.; Beckwith, J.B.; et al. Clinically relevant subsets identified by gene expression patterns support a revised ontogenic model of Wilms tumor: A children's oncology group study. Neoplasia 2012, 14, 742-756. [CrossRef] [PubMed]

10. Fukuzawa, R.; Reeve, A.E. Molecular pathology and epidemiology of nephrogenic rests and Wilms tumors. J. Pediatr. Hematol. Oncol. 2007, 29, 589-594. [CrossRef] [PubMed]

11. Perlman, E.J.; Grundy, P.E.; Anderson, J.R.; Jennings, L.J.; Green, D.M.; Dome, J.S.; Shamberger, R.C.; Ruteshouser, E.C.; Huff, V. WT1 mutation and 11p15 loss of heterozygosity predict relapse in very low-risk Wilms tumors treated with surgery alone: A children's oncology group study. J. Clin. Oncol. 2011, 29, 698-703. [CrossRef] [PubMed]

12. Grundy, P.E.; Telzerow, P.E.; Breslow, N.; Moksness, J.; Huff, V.; Paterson, M.C. Loss of heterozygosity for chromosomes 16q and 1p in wilms' tumors predicts an adverse outcome. Cancer Res. 1994, 54, 2331-2333. [PubMed]

13. Maw, M.A.; Grundy, P.E.; Millow, L.J.; Eccles, M.R.; Dunn, R.S.; Smith, P.J.; Feinberg, A.P.; Law, D.J.; Paterson, M.C.; Telzerow, P.E.; et al. A third Wilms' tumor locus on chromosome 16q. Cancer Res. 1992, 52, 3094-3098. [PubMed]

14. Grundy, R.G.; Pritchard, J.; Scambler, P.; Cowell, J.K. Loss of heterozygosity on chromosome 16 in sporadic Wilms' tumour. Br. J. Cancer 1998, 78, 1181-1187. [CrossRef] [PubMed]

15. Klamt, B.; Schulze, M.; Thate, C.; Mares, J.; Goetz, P.; Kodet, R.; Scheulen, W.; Weirich, A.; Graf, N.; Gessler, M. Allele loss in Wilms tumors of chromosome arms 11q, 16q, and 22q correlate with clinicopathological parameters. Genes Chromosomes Cancer 1998, 22, 287-294. [CrossRef]

16. Wittmann, S.; Zirn, B.; Alkassar, M.; Ambros, P.; Graf, N.; Gessler, M. Loss of 11q and 16q in Wilms tumors is associated with anaplasia, tumor recurrence, and poor prognosis. Genes Chromosomes Cancer 2007, 46, 163-170. [CrossRef] [PubMed]

17. Spreafico, F.; Gamba, B.; Mariani, L.; Collini, P.; D’Angelo, P.; Pession, A.; Di Cataldo, A.; Indolfi, P.; Nantron, M.; Terenziani, M.; et al. Loss of heterozygosity analysis at different chromosome regions in wilms tumor confirms $1 \mathrm{p}$ allelic loss as a marker of worse prognosis: A study from the Italian association of pediatric hematology and oncology. J. Urol. 2013, 189, 260-266. [CrossRef] [PubMed] 
18. Grundy, P.E.; Breslow, N.E.; Li, S.; Perlman, E.; Beckwith, J.B.; Ritchey, M.L.; Shamberger, R.C.; Haase, G.M.; D'Angio, G.J.; Donaldson, M.; et al. Loss of heterozygosity for chromosomes 1p and 16q is an adverse prognostic factor in favorable-histology Wilms tumor: A report from the national Wilms tumor study group. J. Clin. Oncol. 2005, 23, 7312-7321. [CrossRef] [PubMed]

19. Mummert, S.K.; Lobanenkov, V.A.; Feinberg, A.P. Association of chromosome arm 16q loss with loss of imprinting of insulin-like growth factor-II in Wilms tumor. Genes Chromosomes Cancer 2005, 43, 155-161. [CrossRef] [PubMed]

20. Gratias, E.J.; Dome, J.S.; Jennings, L.J.; Chi, Y.Y.; Tian, J.; Anderson, J.; Grundy, P.; Mullen, E.A.; Geller, J.I.; Fernandez, C.V.; et al. Association of chromosome 1q gain with inferior survival in favorable-histology Wilms tumor: A report from the children's oncology group. J. Clin. Oncol. 2016, 34, 3189-3194. [CrossRef] [PubMed]

21. Lu, Y.J.; Hing, S.; Williams, R.; Pinkerton, R.; Shipley, J.; Pritchard-Jones, K.; UK Children's Cancer Study Goup Wilms' tumor group. Chromosome 1q expression profiling and relapse in Wilms' tumour. Lancet 2002, 360, 385-386. [CrossRef]

22. Hing, S.; Lu, Y.J.; Summersgill, B.; King-Underwood, L.; Nicholson, J.; Grundy, P.; Grundy, R.; Gessler, M.; Shipley, J.; Pritchard-Jones, K. Gain of 1q is associated with adverse outcome in favorable histology Wilms' tumors. Am. J. Pathol. 2001, 158, 393-398. [CrossRef]

23. Huang, C.C.; Gadd, S.; Breslow, N.; Cutcliffe, C.; Sredni, S.T.; Helenowski, I.B.; Dome, J.S.; Grundy, P.E.; Green, D.M.; Fritsch, M.K.; et al. Predicting relapse in favorable histology Wilms tumor using gene expression analysis: A report from the renal tumor committee of the children's oncology group. Clin. Cancer Res. 2009, 15, 1770-1778. [CrossRef] [PubMed]

24. Segers, H.; van den Heuvel-Eibrink, M.M.; Williams, R.D.; van Tinteren, H.; Vujanic, G.; Pieters, R.; Pritchard-Jones, K.; Bown, N.; Children's Cancer and Leukaemia Group and the U., K. Cancer Cytogenetics Group. Gain of $1 \mathrm{q}$ is a marker of poor prognosis in Wilms' tumors. Genes Chromosomes Cancer 2013, 52, 1065-1074. [CrossRef] [PubMed]

25. Gratias, E.J.; Jennings, L.J.; Anderson, J.R.; Dome, J.S.; Grundy, P.; Perlman, E.J. Gain of 1q is associated with inferior event-free and overall survival in patients with favorable histology Wilms tumor: A report from the children's oncology group. Cancer 2013, 119, 3887-3894. [CrossRef] [PubMed]

26. Chagtai, T.; Zill, C.; Dainese, L.; Wegert, J.; Savola, S.; Popov, S.; Mifsud, W.; Vujanic, G.; Sebire, N.; Le Bouc, Y.; et al. Gain of $1 \mathrm{q}$ as a prognostic biomarker in Wilms tumors (wts) treated with preoperative chemotherapy in the international society of paediatric oncology (siop) WT 2001 trial: A SIOP renal tumours biology consortium study. J. Clin. Oncol. 2016, 34, 3195-3203. [CrossRef] [PubMed]

27. Kilday, J.P.; Mitra, B.; Domerg, C.; Ward, J.; Andreiuolo, F.; Osteso-Ibanez, T.; Mauguen, A.; Varlet, P.; Le Deley, M.C.; Lowe, J.; et al. Copy number gain of 1q25 predicts poor progression-free survival for pediatric intracranial ependymomas and enables patient risk stratification: A prospective European clinical trial cohort analysis on behalf of the children's cancer leukaemia group (CCLG), societe francaise d'oncologie pediatrique (SFOP), and international society for pediatric oncology (SIOP). Clin. Cancer Res. 2012, 18, 2001-2011. [PubMed]

28. Lo, K.C.; Ma, C.; Bundy, B.N.; Pomeroy, S.L.; Eberhart, C.G.; Cowell, J.K. Gain of 1q is a potential univariate negative prognostic marker for survival in medulloblastoma. Clin. Cancer Res. 2007, 13, 7022-7028. [CrossRef] [PubMed]

29. Pezzolo, A.; Rossi, E.; Gimelli, S.; Parodi, F.; Negri, F.; Conte, M.; Pistorio, A.; Sementa, A.; Pistoia, V.; Zuffardi, O.; et al. Presence of 1q gain and absence of $7 \mathrm{p}$ gain are new predictors of local or metastatic relapse in localized resectable neuroblastoma. Neuro Oncol. 2009, 11, 192-200. [CrossRef] [PubMed]

30. Mackintosh, C.; Ordonez, J.L.; Garcia-Dominguez, D.J.; Sevillano, V.; Llombart-Bosch, A.; Szuhai, K.; Scotlandi, K.; Alberghini, M.; Sciot, R.; Sinnaeve, F.; et al. 1q gain and cdt2 overexpression underlie an aggressive and highly proliferative form of Ewing sarcoma. Oncogene 2012, 31, 1287-1298. [CrossRef] [PubMed]

31. Ooms, A.H.; Gadd, S.; Gerhard, D.S.; Smith, M.A.; Guidry Auvil, J.M.; Meerzaman, D.; Chen, Q.R.; Hsu, C.H.; Yan, C.; Nguyen, C.; et al. Significance of tp53 mutation in Wilms tumors with diffuse anaplasia: A report from the children's oncology group. Clin. Cancer Res. 2016, 22, 5582-5591. [CrossRef] [PubMed] 
32. Bardeesy, N.; Falkoff, D.; Petruzzi, M.J.; Nowak, N.; Zabel, B.; Adam, M.; Aguiar, M.C.; Grundy, P.; Shows, T.; Pelletier, J. Anaplastic Wilms' tumour, a subtype displaying poor prognosis, harbours p53 gene mutations. Nat. Genet. 1994, 7, 91-97. [CrossRef] [PubMed]

33. Gadd, S.; Huff, V.; Walz, A.L.; Ooms, A.; Armstrong, A.E.; Gerhard, D.S.; Smith, M.A.; Auvil, J.M.G.; Meerzaman, D.; Chen, Q.R.; et al. A children's oncology group and target initiative exploring the genetic landscape of Wilms tumor. Nat. Genet. 2017, 49, 1487-1494. [CrossRef] [PubMed]

34. Williams, R.D.; Al-Saadi, R.; Natrajan, R.; Mackay, A.; Chagtai, T.; Little, S.; Hing, S.N.; Fenwick, K.; Ashworth, A.; Grundy, P.; et al. Molecular profiling reveals frequent gain of mycn and anaplasia-specific loss of $4 \mathrm{q}$ and $14 \mathrm{q}$ in wilms tumor. Genes Chromosomes Cancer 2011, 50, 982-995. [CrossRef] [PubMed]

35. Wegert, J.; Vokuhl, C.; Ziegler, B.; Ernestus, K.; Leuschner, I.; Furtwangler, R.; Graf, N.; Gessler, M. Tp53 alterations in Wilms tumour represent progression events with strong intratumour heterogeneity that are closely linked but not limited to anaplasia. J. Pathol. Clin. Res. 2017, 3, 234-248. [CrossRef] [PubMed]

36. Franken, J.; Lerut, E.; Van Poppel, H.; Bogaert, G. P53 immunohistochemistry expression in Wilms tumor: A prognostic tool in the detection of tumor aggressiveness. J. Urol. 2013, 189, 664-670. [CrossRef] [PubMed]

37. Norris, M.D.; Brian, M.J.; Vowels, M.R.; Stewart, B.W. N-myc amplification in Wilms' tumor. Cancer Genet. Cytogenet. 1988, 30, 187-189. [CrossRef]

38. McQuaid, S.; O'Meara, A. N-myc oncogene amplification in paediatric tumours. Ir. J. Med. Sci. 1990, 159, 172-174. [CrossRef] [PubMed]

39. Schaub, R.; Burger, A.; Bausch, D.; Niggli, F.K.; Schafer, B.W.; Betts, D.R. Array comparative genomic hybridization reveals unbalanced gain of the mycn region in Wilms tumors. Cancer Genet. Cytogenet. 2007, 172, 61-65. [CrossRef] [PubMed]

40. Pugh, T.J.; Morozova, O.; Attiyeh, E.F.; Asgharzadeh, S.; Wei, J.S.; Auclair, D.; Carter, S.L.; Cibulskis, K.; Hanna, M.; Kiezun, A.; et al. The genetic landscape of high-risk neuroblastoma. Nat. Genet. 2013, 45, 279-284. [CrossRef] [PubMed]

41. Zirn, B.; Hartmann, O.; Samans, B.; Krause, M.; Wittmann, S.; Mertens, F.; Graf, N.; Eilers, M.; Gessler, M. Expression profiling of Wilms tumors reveals new candidate genes for different clinical parameters. Int. J. Cancer 2006, 118, 1954-1962. [CrossRef] [PubMed]

42. Wittmann, S.; Wunder, C.; Zirn, B.; Furtwangler, R.; Wegert, J.; Graf, N.; Gessler, M. New prognostic markers revealed by evaluation of genes correlated with clinical parameters in Wilms tumors. Genes Chromosomes Cancer 2008, 47, 386-395. [CrossRef] [PubMed]

43. Williams, R.D.; Al-Saadi, R.; Chagtai, T.; Popov, S.; Messahel, B.; Sebire, N.; Gessler, M.; Wegert, J.; Graf, N.; Leuschner, I.; et al. Subtype-specific fbxw7 mutation and mycn copy number gain in Wilms' tumor. Clin. Cancer Res. 2010, 16, 2036-2045. [CrossRef] [PubMed]

44. Williams, R.D.; Chagtai, T.; Alcaide-German, M.; Apps, J.; Wegert, J.; Popov, S.; Vujanic, G.; van Tinteren, H.; van den Heuvel-Eibrink, M.M.; Kool, M.; et al. Multiple mechanisms of mycn dysregulation in Wilms tumour. Oncotarget 2015, 6, 7232-7243. [CrossRef] [PubMed]

45. Green, D.M.; Jaffe, N. The role of chemotherapy in the treatment of Wilms' tumor. Cancer 1979, 44, 52-57. [CrossRef]

46. Green, D.M.; Breslow, N.E.; Beckwith, J.B.; Ritchey, M.L.; Shamberger, R.C.; Haase, G.M.; D'Angio, G.J.; Perlman, E.; Donaldson, M.; Grundy, P.E.; et al. Treatment with nephrectomy only for small, stage I/favorable histology Wilms' tumor: A report from the national Wilms' tumor study group. J. Clin. Oncol. 2001, 19, 3719-3724. [CrossRef] [PubMed]

47. Shamberger, R.C.; Anderson, J.R.; Breslow, N.E.; Perlman, E.J.; Beckwith, J.B.; Ritchey, M.L.; Haase, G.M.; Donaldson, M.; Grundy, P.E.; Weetman, R.; et al. Long-term outcomes for infants with very low risk Wilms tumor treated with surgery alone in national Wilms tumor study-5. Ann. Surg. 2010, 251, 555-558. [CrossRef] [PubMed]

48. Fernandez, C.V.; Perlman, E.J.; Mullen, E.A.; Chi, Y.Y.; Hamilton, T.E.; Gow, K.W.; Ferrer, F.A.; Barnhart, D.C.; Ehrlich, P.F.; Khanna, G.; et al. Clinical outcome and biological predictors of relapse after nephrectomy only for very low-risk Wilms tumor: A report from children's oncology group aren0532. Ann. Surg. 2017, 265, 835-840. [CrossRef] [PubMed] 
49. Dix, D.B.; Fernandez, C.V.; Chi, Y.-Y.; Anderson, J.R.; Mullen, E.A.; Geller, J.I.; Gratias, E.J.; Khanna, G.; Kalapurakal, J.A.; Perlman, E.J.; et al. Augmentation of therapy for favorable-histology Wilms tumor with combined loss of heterozygosity of chromosomes 1p and 16q: A report from the children's oncology group studies AREN0532 and AREN0533. J. Clin. Oncol. 2015, 33, 10009.

50. Fernandez, C.V.; Mullen, E.A.; Chi, Y.Y.; Ehrlich, P.F.; Perlman, E.J.; Kalapurakal, J.A.; Khanna, G.; Paulino, A.C.; Hamilton, T.E.; Gow, K.W.; et al. Outcome and prognostic factors in stage III favorable-histology Wilms tumor: A report from the children's oncology group study AREN0532. J. Clin. Oncol. 2018, 36, 254-261. [CrossRef] [PubMed]

51. Dix, D.B.; Seibel, N.L.; Chi, Y.Y.; Khanna, G.; Gratias, E.; Anderson, J.R.; Mullen, E.A.; Geller, J.I.; Kalapurakal, J.A.; Paulino, A.C.; et al. Treatment of stage iv favorable histology Wilms tumor with lung metastases: A report from the children's oncology group AREN0533 study. J. Clin. Oncol. 2018, 36, 1564-1570. [CrossRef] [PubMed]

52. Daw, N.; Anderson, J.; Kalapurakal, J.A.; Hoffer, F.; Geller, J.; Perlman, E.; Ehrlich, P.; Mullen, E.; Warwick, A.; Grundy, P.; et al. Treatment of Stage II-IV Diffuse Anaplastic Wilms Tumor: Results from the Children's Oncology Group AREN0321 Study. In Pediatric Blood \& Cancer, Proceedings of the 46th Congress of the International Society of Paediatric Oncology (SIOP), Toronto, ON, Canada, 22-25 October 2014; Wiley-Blackwell: Hoboken, NJ, USA; Volume 61, pp. S11-S13.

53. Ehrlich, P.; Chi, Y.Y.; Chintagumpala, M.M.; Hoffer, F.A.; Perlman, E.J.; Kalapurakal, J.A.; Warwick, A.; Shamberger, R.C.; Khanna, G.; Hamilton, T.E.; et al. Results of the first prospective multi-institutional treatment study in children with bilateral Wilms tumor (aren0534): A report from the children's oncology group. Ann. Surg. 2017, 266, 470-478. [CrossRef] [PubMed]

54. Verschuur, A.; Van Tinteren, H.; Graf, N.; Bergeron, C.; Sandstedt, B.; de Kraker, J. Treatment of pulmonary metastases in children with stage IV nephroblastoma with risk-based use of pulmonary radiotherapy. J. Clin. Oncol. 2012, 30, 3533-3539. [CrossRef] [PubMed]

55. Ehrlich, P.F.; Ferrer, F.A.; Ritchey, M.L.; Anderson, J.R.; Green, D.M.; Grundy, P.E.; Dome, J.S.; Kalapurakal, J.A.; Perlman, E.J.; Shamberger, R.C. Hepatic metastasis at diagnosis in patients with Wilms tumor is not an independent adverse prognostic factor for stage iv Wilms tumor: A report from the children's oncology group/national Wilms tumor study group. Ann. Surg. 2009, 250, 642-648. [CrossRef] [PubMed]

56. Daw, N.C.; Anderson, J.R.; Hoffer, F.A.; Geller, J.I.; Kalapurakal, J.A.; Perlman, E.J.; Ehrlich, P.F.; Mullen, E.A.; Gratias, E.J.; Grundy, P.E.; et al. A phase 2 study of vincristine and irinotecan in metastatic diffuse anaplastic Wilms tumor: Results from the children's oncology group AREN0321 study. J. Clin. Oncol. 2014, 32, 10032.

57. Cresswell, G.D.; Apps, J.R.; Chagtai, T.; Mifsud, B.; Bentley, C.C.; Maschietto, M.; Popov, S.D.; Weeks, M.E.; Olsen, O.E.; Sebire, N.J.; et al. Intra-tumor genetic heterogeneity in Wilms tumor: Clonal evolution and clinical implications. EBioMedicine 2016, 9, 120-129. [CrossRef] [PubMed]

58. Dome, J.S.; Graf, N.; Geller, J.I.; Fernandez, C.V.; Mullen, E.A.; Spreafico, F.; Van den Heuvel-Eibrink, M.; Pritchard-Jones, K. Advances in Wilms tumor treatment and biology: Progress through international collaboration. J. Clin. Oncol. 2015, 33, 2999-3007. [CrossRef] [PubMed]

(C) 2018 by the authors. Licensee MDPI, Basel, Switzerland. This article is an open access article distributed under the terms and conditions of the Creative Commons Attribution (CC BY) license (http:/ / creativecommons.org/licenses/by/4.0/). 